\title{
List of families
}

This is an alphabetical list of nobles' patronymic names that correspond with the archival fonds used in primary research. Normally the fonds is named after the family or individual who produced it. Some fonds are named after a château and contain records for several families; patronymic names have been included in this list for families continuing to produce records after 1789 . The province of origin and the type of nobility are given. Where applicable admission to honours of the court and/or membership of the Association d'entraide de la noblesse française (ANF) are also mentioned.

To identify nobles' patronymic names I used the following reference works that contain information on coats of arms and documentary proofs of nobility: Gaston Saffroy, Bibliographie généalogique, héraldique et nobiliaire de la France 5 vols (Paris, 1974); Vicomte Albert Révérend, Les Familles titrées et anoblies au XIXe siècle (Paris, 1974); Louis d'IzarnyGargas, Jean-Jacques Lartigue, and Jean de Vaulchier, Nouveau nobiliaire de France 3 vols (Versailles, 1997); François Bluche, Les Honneurs de la cour 2 ed. (Paris, 2000); Jean de Vaulchier, Jacques Amable de Saulieu, and Jean de Bodinat, Armorial de l'ANF (Paris, 2004); Régis Valette, Catalogue de la noblesse française (Paris, 2007).

Abbadie (d') Béarn, extraction Albertas (d') Provence, extraction, ANF

Alexandry d'Orengiani (d')

Savoie, extraction, ANF
Andlau (d') Alsace, extraction, honneurs de la cour, ANF

Apchier (d') Auvergne, extraction, honneurs de la cour 
Arlot de Saint-Saud (d') Périgord, extraction, ANF

Baillon (de) Paris, par charge

Bayly (de) Périgord, extraction, honneurs de la cour

Beaumont-Beynac (de) Périgord, extraction, honneurs de la cour, ANF

Belot (de) Blésois, par charge, ANF

Bigot de la Touanne Berry, par charge, ANF

Bizemont (de) Orléanais, extraction, ANF

Blacas d'Aulps (de) Provence, extraction, ANF

Bodard de la Jacopière Anjou, par lettres 19th century, ANF

Bonald (de) Rouergue, extraction, ANF

Bonnin de La Bonninière de Beaumont Touraine, extraction, honneurs de la cour, ANF

Bot du Grégo (du) Bretagne, extraction

Botherel (de) Bretagne, extraction, honneurs de la cour, ANF

Briet de Rainvillers Picardie, par charge, ANF

Brillet de Candé Anjou, extraction, honneurs de la cour

Brochard de La Rochebrochard Poitou, extraction, ANF

Brun de Montesquiou (de)

Gévaudan, extraction

Brunet de Castelpers de Panat

(de) Roussillon, extraction
Buisson de Bournazel (de)

Rouergue, extraction

Callières (de) Saintonge, extraction

Camy (de) Quercy, extraction

Carbonnières (de) Limousin, extraction, honneurs de la cour, ANF

Cardon de Sandrans (de) Lyonnais, par charge

Castellane (de) Provence, extraction, honneurs de la cour, ANF

Chalvet de Rochemonteix Auvergne, extraction

Chambrun (de) Bourbonnais, par charge

Chanaleilles (de) Vivarais, extraction, honneurs de la cour

Chapel d'Espinassoux (de) Gévaudan, par lettres 19th century

Chastel de Servières (de) Gévaudan, extraction

Châteauneuf-Randon du Tournel (de) Gévaudan, extraction, honneurs de la cour, ANF

Chaudruc de Crazannes Saintonge, par charge

Chauvelin (de) Vendômois, par charge, honneurs de la cour

Chazelles (de) Languedoc, par lettres 19th century

Chevigné (de) Bretagne, extraction, honneurs de la cour, ANF

Choiseul (de) Champagne, extraction, honneurs de la cour, ANF 
Clermont-Tonnerre (de)

Dauphiné, extraction, honneurs de la cour, ANF

Comarmond (de) Lyonnais, par charge

Coniac (de) Bretagne, par lettres, ANF

Couëssin Bretagne, extraction, ANF

Courson (de) Bretagne, extraction, ANF

Courtin de Neufbourg Forez, lettres de relief de dérogeance, ANF

Croÿ (de) Picardie/Hainaut, extraction, honneurs de la cour, ANF

Cugnac (de) Périgord, extraction, honneurs de la cour, ANF

Dampierre (de) Picardie, extraction, ANF

Decazes Guyenne, par lettres 19th century, ANF

Dondel de Kergonano Bretagne, extraction

Dufour Normandie, par charge

Durfort Civrac de Lorge (de) Languedoc, extraction, honneurs de la cour, ANF

Du Teil Velay, par lettres 19th century

Emé de Marcieu Dauphiné, extraction, honneurs de la cour, ANF

Espagnet (d') Provence, par charge, ANF

Espivent de la Villeboisnet Bretagne, extraction, ANF
Estienne de Saint-Jean (d')

Provence, par charge

Estourmel (d') Picardie, extraction, honneurs de la cour, ANF

Fabre (de) Languedoc, par lettres 19th century, ANF

Faramond (de) Rouergue, extraction, ANF

Faure (de) Roussillon, Capitoul de Toulouse, ANF

Fay (de) Normandie, extraction

Finiels (de) Languedoc, par charge

Fontaines (de) Normandie, extraction, ANF

Fontenay (de) Normandie, extraction

Forbin (de) Provence, extraction, honneurs de la cour, ANF

Forcade (de) États de Béarn

Foresta (de) Provence, extraction, ANF

Foucault (de) Berry, par charge

Framond (de) Gévaudan, extraction

Francheville (de) Bretagne, Scottish origin, recognised noble in France, ANF

Freslon de la Freslonnière (de) Bretagne, extraction, honneurs de la cour, ANF

Fresne de Virel (du) Bretagne, extraction, ANF

Fretard-d'Ecoyeux (de) Angoumois, extraction

Froissard de Broissia (de) Franche-Comté, par lettres, ANF 
Frotier de La Messelière Poitou, extraction, ANF

Galard de Béarn (de) Gascogne, extraction, honneurs de la cour, ANF

Gallet (de) Normandie, extraction

Geouffre de La Pradelle (de) Limousin, par charge

Gérard (de) Périgord, extraction, ANF

Gilbert Touraine, par charge, ANF

Gontaut-Biron (de) Périgord, extraction, ANF

Gontier Toulousain, Capitoul de Toulouse

Gouvello de Keriaval (de)

Bretagne, extraction, honneurs de la cour, ANF

Goyon de Feltre Gascogne, par charge

Grailly (de) Guyenne, extraction, ANF

Grasse (de) Provence, extraction, honneurs de la cour

Grassis Savoie, par lettres

Guillard Paris, par charge

Guyon de Montlivault Orléanais, par lettres, ANF

Hautpoul (d') Languedoc, extraction

Hédouville (de) Île de France, extraction, ANF

Hüe de la Blanche Forez, extraction

Isoard-Vauvenargues (d') Provence, par charge
Jaucourt (de) Bourgogne, extraction, honneurs de la cour

Kersaint (de) Bretagne, extraction, ANF

La Baume-Le Blanc Touraine, extraction, honneurs de la cour

La Borie de Campagne (de) Périgord, par charge, ANF

La Bourdonnaye (de) Bretagne, extraction, honneurs de la cour, ANF

La Croix de Chevrières Dauphiné, par charge, ANF

La Forest d'Armaillé (de) Bretagne, extraction, ANF

La Forest Divonne (de) Savoie, extraction, honneurs de la cour, ANF

La Haye (de) Bretagne, extraction

La Houssaye (de) Normandie, extraction

La Monneraye (de) Bretagne, par lettres, ANF

La Motte (de) Bretagne, extraction

Langlade du Chayla (de) Gévaudan, extraction

Langle (de) Bretagne, extraction, ANF

La Rochefoucauld (de) Angoumois, extraction, honneurs de la cour, ANF

La Rochenégly de Chayla (de) Gévaudan, extraction

Laugier de Beaucouse Provence, extraction 
Le Borgne de Boigne Savoie, par lettres 19th century, ANF

Le Chartier de Sédouy

Normandie, par charge, ANF

Le Chauff de Kerguénec Bretagne, extraction

Lecoq de Boisbaudran Angoumois, par charge

Le Féron Normandie, par charge

Le Forestier Normandie, extraction

Le Fournier Normandie, par lettres

Le Gonidec de Traissan Bretagne, extraction, ANF

Le Gouvello du Timat Bretagne, extraction

Le Guennec Bretagne, extraction

Lenfernat (de) Bourgogne, extraction

Le Ray d'Abrantès Paris, par lettres 19th century

Le Roy Normandie, par lettres 19th century

L'Escale (de) Lorraine, extraction, ANF

Lespinasse (de) Normandie, extraction

L'Estourbeillon (de) Bretagne, extraction

Le Tellier de Louvois Paris, par charge, honneurs de la cour

Le Veneur de Tillières

Normandie, extraction, honneurs de la cour

Lévesque de Puyberneau Poitou, extraction

Lévis Mirepoix (de) Languedoc, extraction, honneurs de la cour, ANF
Lezay de Marnesia (de) FrancheComté, extraction, honneurs de la cour

Liebhaber (de) Saxony, French naturalisation

Luppé (de) Armagnac, extraction, honneurs de la cour, ANF

Mackau (de) Alsace, Irish origin recognised noble in France

Madaillan Périgord, lettres de relief de dérogeance, $\mathrm{ANF}$

Mahuet (de) Lorraine, par lettres

Maillard de la Gournerie Bretagne, extraction

Maison Paris, par lettres 19th century

Maréchal de Longeville FrancheComté, par charge

Marescot (de) Normandie, par lettres

Marne (de) Lorraine, extraction

Marÿe de Marigny Poitou, par charge

Massol (de) Bourgogne, par charge, ANF

Metz-Noblat (de) Lorraine, extraction, ANF

Milleville Orléanais, par charge

Molette de Morangiès (de) Auvergne, extraction, honneurs de la cour, ANF

Montrichard (de) FrancheComté, extraction, ANF

Moré de Charaix (de) Gévaudan, extraction

Morel (de) Picardie, par lettres

Morel de La Colombe Auvergne, extraction, ANF 
Moucheron (de) Normandie, extraction, ANF

Mullot de Villenaut (de)

Nivernais, extraction

Murat Quercy, par lettres 19th century, ANF

Nagu (de) Beaujolais, extraction, honneurs de la cour

Narbonne-Lara (de) Languedoc, extraction, honneurs de la cour

Nettancourt (de) Champagne/ Lorraine, extraction, honneurs de la cour

Orillard de Villemanzy Touraine, par lettres 19 th century

\section{Passemar de Saint-André} Languedoc, extraction, ANF

Perrochel de Grandchamp (de) Maine, par charge, ANF Pilliers (des) Lorraine, extraction Pradier d'Agrain (de) Velay/ Bourgogne, par lettres, ANF

Quatrebarbes (de) Anjou, extraction, honneurs de la cour, ANF

Quentin Normandie, par lettres Quifistre de Bavallan Bretagne, extraction, honneurs de la cour

Quillebeuf (de) Normandie, par lettres, 19th century

Raymond (de) Rouergue, ANF

Reinach (de) Haut Alsace, extraction, ANF
Ripert d'Alauzier Comtat Venaissin, extraction, ANF

Rochechouart de Mortemart (de) Limousin, extraction, honneurs de la cour, ANF

Rolland du Noday Bretagne, extraction, ANF

Rozières (de) Lorraine, par lettres, ANF

Sade (de) Comtat Venaissin, extraction, honneurs de la cour, ANF

Saint-Astier (de) Périgord, extraction, honneurs de la cour

Saint-Blimond (de) Picardie, extraction

Saint-Exupéry (de) Périgord, extraction, honneurs de la cour, ANF

Salmon de Loiray (de) Orléanais, extraction

Sanzillon (de) Périgord, extraction Sarcus (de) Picardie, extraction, ANF

Sesmaisons (de) Bretagne, extraction, honneurs de la cour, ANF

Suremain (de) Bourgogne, par charge

Taillepied Île de France, par charge, ANF

Taillevis de Perrigny Blésois, extraction

Talleyrand-Périgord (de) Périgord, extraction, honneurs de la cour, ANF

Teillard Auvergne, extraction 
Teillard Rancilhac de Chazelles

Auvergne, par lettres, 19th century

Théas de Caille de Thorenc (de) Provence, aggregated

Torrilhon de Vacherolles (de)

Velay, par charge

Tournemire (de) Auvergne, extraction, honneurs de la cour, ANF

Tournier (de) Toulousain, Capitoul de Toulouse

Tournu de Ventavon Dauphiné, par charge

Tredecini de Saint-Séverin Savoie

Treil (de) Languedoc, extraction

Vacher de Tournemire Auvergne, par lettres, 19 th century

Vassal (de) Quercy, extraction
Vaulchier (de) Franche-Comté, par charge

Veyre de Soras (de) Vivarais, par charge, ANF

Vigneral (de) Normandie, par lettres

Villardi de Montlaur (de) Comtat Venaissin, extraction, ANF

Villeneuve (Trans, Flayosc, Bargemon, Esclapon) (de) Provence, extraction, honneurs de la cour, ANF

Vincent Lyonnais, par charge

Vintimille du Luc (de) Provence, extraction, honneurs de la cour

Vogüé (de) Vivarais, extraction, honneurs de la cour, ANF

Widranges (de) Lorraine, extraction 